\title{
Itinéraires Itinéraires
}

Littérature, textes, cultures

2019-2 et $3 \mid 2019$

Corps masculins et nation : textes, images, représentations

\section{Spencer Tracy et la reconfiguration de la masculinité hégémonique américaine après la Grande Dépression}

Spencer Tracy and the Reconfiguration of American Hegemonic Masculinity

after the Great Depression

Jules Sandeau

\section{OpenEdition}

Journals

Édition électronique

URL : http://journals.openedition.org/itineraires/6812

DOI : $10.4000 /$ itineraires.6812

ISSN : 2427-920X

Éditeur

Pléiade

Référence électronique

Jules Sandeau, «Spencer Tracy et la reconfiguration de la masculinité hégémonique américaine après la Grande Dépression », Itinéraires [En ligne], 2019-2 et 3 | 2019, mis en ligne le 27 novembre 2019, consulté le 15 décembre 2019. URL : http://journals.openedition.org/itineraires/6812 ; DOI : 10.4000/ itineraires.6812

Ce document a été généré automatiquement le 15 décembre 2019.

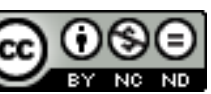

Itinéraires est mis à disposition selon les termes de la licence Creative Commons Attribution - Pas d'Utilisation Commerciale - Pas de Modification 4.0 International. 


\title{
Spencer Tracy et la reconfiguration de la masculinité hégémonique américaine après la Grande Dépression
}

\author{
Spencer Tracy and the Reconfiguration of American Hegemonic Masculinity \\ after the Great Depression
}

Jules Sandeau

Si Spencer Tracy avait déjà acquis une certaine renommée pendant la première moitié des années 1930 (Loew 2008), notamment grâce à sa prestation dans The Power and the Glory (William K. Howard, 1933) qui reçut un très bon accueil critique (Curtis 2011:200, 208-210), ce sont ses rôles dans Fury (Fritz Lang, 1936) et San Francisco (W. S. Van Dyke, 1936) qui lui permirent de devenir une star hollywoodienne de premier plan au milieu de la décennie. Suite à ces deux films, l'acteur reçoit plus de 3000 courriers de fans par mois, alors qu'il n'en recevait pas plus de 200 par mois auparavant ${ }^{1}$. Sa prestation dans Captains Courageous (Victor Fleming, 1937) renforce sa popularité et lui permet de remporter un Academy Award. Il triomphe ensuite régulièrement au box-office jusqu'au tournant des années 1940 dans des films tels que Test Pilot (Victor Fleming, 1938), Boys Town (Norman Taurog, 1938) - pour lequel il remporte un second Oscar -, Northwest Passage (King Vidor, 1940) ou Boom Town (Jack Conway, 1940). À partir de 1938, il figure régulièrement dans le « Top Ten Box Office Star » établi par Quigley sur la base d'un sondage réalisé auprès des exploitants (Steinberg 1980: 57-58), et les enquêtes menées par George Gallup en 1940 révèlent qu'il est alors la star hollywoodienne la plus appréciée du public états-unien, loin devant Mickey Rooney et Clark Gable, les deux stars les plus populaires après lui (Gallup 1979).

2 Cet article se propose d'éclairer les raisons du succès exceptionnel de Spencer Tracy durant la seconde moitié des années 1930 en replaçant son image dans le contexte idéologique états-unien de l'époque. Selon Richard Dyer ([1979] 2004a : 29), les stars expriment et travaillent des contradictions idéologiques qu'elles "gèrent» ou 
« résolvent » dans le domaine de l'imaginaire. Loin d'être univoques, les images de stars se caractérisent souvent par une grande complexité que Dyer analyse grâce au concept de "polysémie structurée », en soulignant la multiplicité des "textes médiatiques " (films, articles de magazine, photos promotionnelles, etc.) qui convergent pour constituer la persona d'une star particulière (Dyer [1979] 2004a: 62-69). Si cette polysémie peut être source de tensions entre différentes dimensions de la persona de la star, qui manifeste souvent une certaine incohérence, elle contribue également à la popularité des stars dans la mesure où différents publics peuvent ainsi sélectionner et s'approprier les aspects de l'image qui leur parlent le plus (Dyer [1986] 2004b: 4). Spencer Tracy en est un parfait exemple, comme en témoigne le fait qu'il séduise un public très hétérogène (les hommes comme les femmes, les membres des couches populaires comme ceux de la classe supérieure, les habitants des petites communautés comme ceux des grandes agglomérations, etc.). Les sondages réalisés par Gallup en avril 1940 montrent par exemple qu'il est non seulement la star la plus populaire auprès des hommes, mais également l'acteur masculin le plus apprécié des femmes, devant Clark Gable, Tyrone Power ou Charles Boyer qui, contrairement à lui, ont pourtant une image de séducteur (Gallup 1979).

3 Je me propose ici de mettre en lumière la complexité de la persona de Tracy en me focalisant sur sa dimension genrée et en analysant aussi bien les rôles qu'il tient dans ses films à succès de la seconde moitié des années 1930, que les discours dont il fait l'objet dans la presse états-unienne. Il s'agira de montrer comment l'image de l'acteur résout « magiquement » des " contradictions internes à l'idéologie dominante » (Dyer [1979] 2004a : 21) en cette période où la "masculinité hégémonique » américaine se réajuste suite à la "perturbation de l'ordre de genre » suscitée par la Dépression (Connell $2014:$ 74-75, 84). Le processus de reconstruction nationale qui s'opère alors dont témoignent entre autres l'usage exponentiel de l'expression "American Way » à partir du milieu de la décennie (Wall 2009: 15-33) ou la récurrence avec laquelle Hollywood rend alors hommage aux valeurs américaines et revisite l'histoire du pays ${ }^{2}$ -, s'articule en effet à un retour à l'ordre patriarcal en réaction à la fragilisation momentanée des rapports de genre conventionnels générée par la crise (Dhommée 2002 : 275-319). Dans un contexte où les stars masculines dominent à nouveau le box-office et où les stars étrangères peinent à conserver leur popularité (310-314), le succès de Tracy est emblématique de ce recentrement du cinéma hollywoodien sur la masculinité américaine.

4 Malgré un père d'origine irlandaise, l'acteur est en effet construit avec insistance comme une incarnation exemplaire de l'identité américaine. Au début de la série d'articles qu'il lui consacre en 1937, le magazine Photoplay raconte que le jeune Spencer a grandi dans une maison "solidement américaine » et s'est engagé pour servir sous les drapeaux dès l'âge de 17 ans $^{3}$. S'il joue un pêcheur luso-américain dans Captains Courageous et des prêtres catholiques irlando-américains dans San Francisco et Boys Town, l'ethnicité de ces personnages ne compromet en rien leur intégration à la nation américaine dont ils incarnent les valeurs. Cette dimension de son image se renforce à la fin de la décennie, notamment grâce à ses rôles dans Test Pilot et Boom Town, et même dans Stanley and Livingstone (Henry King, 1939) qui, même s'il met en scène la réconciliation de son personnage avec ses origines britanniques, le construit avec insistance comme un Américain dont la mission civilisatrice est comparée à la conquête de l'Ouest. De même, tandis que Paul Muni rencontre le succès dans les rôles de Louis Pasteur et d'Émile Zola, Tracy incarne quant à lui des grandes figures de l'histoire 
américaine : le Major Rogers dans Northwest Passage ou Thomas Edison dans Edison, the Man (Clarence Brown, 1940).

5 Afin de souligner les enjeux socio-culturels qui sous-tendent la persona de Tracy et d'éclairer les raisons de sa popularité, cet article replace la masculinité américaine incarnée par l'acteur dans le contexte idéologique de la seconde moitié des années 1930. Il s'agit notamment de montrer que l'image de Tracy pendant cette période ne peut pleinement être comprise qu'en étant réinscrite à la fois dans l'histoire à court terme de la reconstruction nationale et masculine qui fit suite à la Dépression, et dans l'histoire à long terme de la reconfiguration de la masculinité hégémonique américaine depuis la fin du xix ${ }^{\mathrm{e}}$ siècle.

\section{« Un puissant Phœnix » : reconstruction nationale et remasculinisation}

6 À plusieurs égards, l'image de Tracy est emblématique des différentes «stratégies de remasculinisation» (Armengol 2014: 60-61) qui visent alors à exorciser les perturbations de l'ordre de genre générées par la crise. La Dépression a en effet pour conséquence une fragilisation du pouvoir économique des hommes dans un certain nombre de familles de classe moyenne et ouvrière. Les femmes sont souvent obligées de travailler pour compenser l'absence ou la baisse de salaire de leur mari, et deviennent le centre de l'autorité au sein du foyer (Mintz 1988: 138 ; Dhommée 2002: 247). Cette reconfiguration temporaire des rapports de genre s'accompagne d'un sentiment d'« émasculation » chez des hommes privés de leur travail et donc de leur statut de " breadwinner » (Kimmel [1996] 2012 : 144-146; Hanson 2008 : 110). Les enquêtes réalisées pendant la période témoignent des conséquences du chômage sur la manière dont les hommes sont perçus par leurs proches et se perçoivent eux-mêmes (Komarovsky 1971; Baxandall 1995 : 210-213). Certains ne supportent pas d'être ainsi dépossédés de leur position hégémonique et quittent le foyer, tandis que d'autres se retrouvent assignés à une sphère domestique culturellement perçue comme féminine et participent même parfois aux tâches ménagères malgré le regard des voisins (Filene [1974] 1998: 166; Dhommée $2002: 247$, McElvaine [1984] $2009: 180$, Olszowka $2014: 168$ ). Des productions hollywoodiennes dramatisent le sentiment de perte de masculinité éprouvé par de nombreux Américains en proposant souvent des représentations d'hommes «en crise ", alcooliques, malades, ou plus largement impuissants ou défaillants. Tandis que les stars féminines dominent le box-office, Wallace Beery devient par exemple une star de premier plan en incarnant un pêcheur idiot et alcoolique face à Marie Dressler dans Min and Bill (George W. Hill, 1930), avant de gagner un Oscar pour son rôle de boxeur déchu dans The Champ (King Vidor, 1931) qui se termine sur la mort de son personnage d'homme immature et sans avenir, incapable d'élever son fils correctement du fait de son penchant pour le jeu et la boisson.

7 En réaction à ces bouleversements sociaux, un certain nombre de discours et de représentations témoignent d'une entreprise de «remasculinisation» de l'homme américain à laquelle participe la persona de Tracy. Dans un contexte économique où les idéaux qui valorisent un accomplissement masculin sur le marché du travail sont mis à mal, la masculinité tend à être associée à des qualités possédées par certains individus indépendamment de leur succès dans la sphère professionnelle (Armengol 2014: 60-61), lequel est au cœur de l'idéal du self-made-man. Au milieu de la décennie, les 
psychologues Terman et Miles mettent au point un « test M-F » qui vise à mesurer « la masculinité et la féminité mentales » des individus grâce à un questionnaire sur leurs goûts, leurs connaissances et leurs comportements (Kimmel [1996] 2012: 150-152, Penner 2011 : 40-49). Le projet normatif de ces scientifiques transparait clairement dans leur ouvrage Sex and Personality (1936) qui analyse les résultats des enquêtes menées à partir de ces tests. Seuls les hommes présentant un "déficit de masculinité " y sont considérés comme un problème, tandis que les « effets potentiellement négatifs de l'hypermasculinité » ne sont pas envisagés, signe que cette dernière est perçue par les psychologues comme "un avantage culturel et une qualité sociale » qui pourra contribuer à sortir le pays de la crise (Penner 2011: 44). De même, John L. Griffith déclare alors, dans ses éditos pour le magazine viriliste Athletic Journal, que la Dépression doit être vue comme un "défi à relever» auquel seuls survivront les hommes « forts » et « combatifs » (Pendergast $1997: 61$ ).

Présenté par Photoplay comme un «Irlandais combatif qui a lutté contre la pauvreté et la tragédie - et a gagné ${ }^{-}$» dans un article de 1937 qui retrace la vie de l'acteur, Spencer Tracy peut être vu comme une incarnation emblématique de cette masculinité virile essentialisée. Un fan commence ainsi son éloge en le présentant comme «un homme plein de force et de courage ${ }^{5}$ ", tandis qu'une journaliste de Modern Screen cite le réalisateur W. S. Van Dyke qui déclare à propos de Tracy : "Il est robuste. Tout chez lui est masculin. Il est dur et coriace ; c'est un homme ${ }^{6}$. » L'évocation de ses origines irlandaises sert souvent à enraciner ses traits de personnalité culturellement associés au masculin (agressivité, combativité, courage, etc. ${ }^{7}$ ) dans un tempérament qu'il aurait hérité de ses ancêtres ${ }^{8}$. La naturalisation de sa masculinité passe également par des récits qui reviennent sur son enfance en expliquant qu'il a toujours été un garçon turbulent et "dur à cuire ${ }^{9}$ ». Un article raconte même que, dès sa naissance, le petit Spencer possédait un corps trapu, des bras costauds et un tempérament agressif ${ }^{10}$. La plupart de ses films de la seconde moitié des années 1930 mettent en avant son physique robuste, notamment dans des scènes de bagarre où il a le plus souvent le dessus sur son adversaire. Lors de sa première apparition dans San Francisco, il terrasse ainsi Clark Gable lors d'un combat de boxe, et les deux stars s'affronteront à nouveau dans une longue scène de Boom Town qui insiste sur leur capacité à encaisser les coups jusqu'au sang. Northwest Passage est probablement le film qui insiste le plus sur la robustesse et l'endurance de Tracy qui tient ici le rôle d'un chef militaire à l'énergie inépuisable.

Cette mise en avant de la «dureté » du corps de l'acteur participe de la stratégie la plus "évidente" de "remasculinisation" de l'homme américain touché par la crise: le « retour au corps » (Armengol 2014: 60-61). Si l'importance prise par la vigueur et la force physiques dans la définition de la masculinité américaine ne date pas de la Dépression mais remonte à la fin du XIX ${ }^{\mathrm{e}}$ siècle (Rotundo 1993 : 222-224, Kimmel [1996] 2012 : 89), le succès non démenti de Charles Atlas pendant les années 1930 confirme l'adéquation de l'idéal incarné par ce bodybuilder à un contexte de fragilisation momentanée des identités et rapports de genre conventionnels (Kimmel [1996] 2012 : 152-153). Parmi les incarnations emblématiques de cette masculinité athlétique et musclée, on peut notamment citer le personnage de Superman qui fait son apparition sur les pages illustrées des comics américains pendant la décennie (Kimmel [1996] 2012 : 153-154), ou encore le Tarzan personnifié par Johnny Weissmuller dans les films produit par la MGM suite au succès de Tarzan the Ape Man (W. S. Van Dyke, 1932). Dans la mesure où le corps masculin blanc est très rarement exhibé à l'écran du fait d'un 
imaginaire qui associe la blanchité ${ }^{11}$ à la spiritualité en renvoyant la "différence " raciale du côté de l'animalité et du corps, on peut interpréter la semi-nudité de Tarzan comme une stratégie de «dernier recours » par laquelle le patriarcat blanc tente de naturaliser une domination sociale alors fragilisée en lui donnant un fondement biologique (Dyer 1997 : 146-147) ${ }^{12}$.

Mais ce sont probablement les œuvres d'art - notamment les fresques murales produites dans le cadre du New Deal qui témoignent le plus clairement de la manière dont la muscularité masculine est alors utilisée comme un antidote à la crise. Sur un grand nombre d'entre elles, des ouvriers hypermasculins sont représentés en plein travail, les outils à la main et le torse souvent dénudé. Selon Barbara Melosh (1993 : 155-181), cette "iconographie du travailleur viril» s'enracine entre autres dans l'imaginaire du mouvement syndicaliste américain, et James Penner (2011:29) insiste sur l'importance particulière que prit cette figure ouvrière idéalisée dans la rhétorique de la gauche américaine pendant la Dépression. Si James Cagney est probablement l'incarnation hollywoodienne la plus emblématique de cette virilité prolétarienne, Spencer Tracy s'en rapproche également par ses rôles dans des films comme Riffraff (J. Walter Ruben, 1936), Big City (Frank Borzage, 1937) ou Captains Courageous. Ce dernier se termine d'ailleurs sur l'image d'une statue érigée en hommage aux pêcheurs qui, comme Manuel (Tracy), sont morts en mer en accomplissant leur travail. Le mélange de force et de vulnérabilité qui se dégage de cette sculpture, proche en cela de nombreuses représentations des ouvriers pendant la période (Doss 1997 : 60-64 ; Armengol 2014 : 63), se retrouve chez le personnage de Manuel. En effet, même si son physique robuste et endurant est opposé à celui des membres de la classe supérieure, incarnés par des acteurs plus sveltes (Freddie Bartholomew et Melvyn Douglas), la scène de sa mort insiste sur la vulnérabilité de son corps, qui est littéralement broyé avant de couler au fond de l'océan.

11 Le destin tragique de ce personnage, dont on retrouve un équivalent dans Test Pilot, reste cependant rare chez l'acteur, qui incarne plutôt des hommes surmontant des épreuves et des moments de doute grâce à leur détermination et leur ténacité (Boys Town, Northwest Passage, Edison, the Man, Boom Town, etc.). À l'image du final de San Francisco où les survivants du tremblement de terre de 1906 entonnent d'une seule voix le chant patriotique The Battle Hymn of the Republic en marchant avec résolution vers leur ville en ruines pour la reconstruire pierre par pierre, Tracy personnifie l'espoir d'un renouveau. Les magazines le dépeignent ainsi comme un homme qui a fait des erreurs et a été confronté à des épreuves difficiles, mais qui a trouvé en lui la «force » de les surmonter ${ }^{13}$. Ses échecs lors de ses débuts sur les planches, la maladie de son fils, ses problèmes de couple suite à son aventure avec Loretta Young, ou encore son alcoolisme, sont régulièrement évoqués pour souligner sa capacité à « renaître de ses cendres» tel un "puissant Phœnix ${ }^{14} »$. Dans plusieurs articles, l'acteur s'adresse directement aux lecteurs/trices des fan magazines pour leur expliquer que tout homme peut trouver en lui-même les ressources pour ressortir victorieux des combats intérieurs qu'il doit mener ${ }^{15}$, et que, à l'image de Thomas Edison qu'il incarne à l'écran en 1940, les personnes qui « ne baissent jamais les bras » finiront forcément par réussir, "quels que soient les obstacles qui se dressent en travers de leur route", car le " succès » vient forcément aux gens qui «ne peuvent pas être découragés ${ }^{16}$ ". Ajoutée à la variété des rôles interprétés par l'acteur en termes d'appartenance de classe, l'abstraction de ces discours sur l'importance de la persévérance explique la popularité exceptionnelle de Tracy auprès de toutes les classes sociales, là où la persona d'autres 
stars comme Joan Crawford ou James Cagney en font avant tout une inspiration pour le public des couches populaires pendant la période (Gallup 1979).

En somme, Tracy incarne un homme dont les qualités «masculines» (force, persévérance, courage, endurance, etc.) vont permettre au pays de sortir de la crise, et constitue ainsi un antidote au sentiment d'émasculation et de déroute éprouvé par de nombreux hommes durant les années les plus noires de la Dépression. Au-delà de cet idéal masculin, Tracy personnifie aussi probablement la nation tout entière qui, à l'image de la star, doit alors renaître de ses cendres.

\section{Nature, naturel et authenticité}

13 Loin de ne reposer que sur un ensemble de qualités "masculines ", la régénération masculine et nationale incarnée par Tracy prend place dans un univers spécifique. Celui-ci se caractérise d'abord par une omniprésence des hommes. La plupart des films de l'acteur à cette période tournent en effet autour d'une amitié masculine (Test Pilot, Boom Town, etc.) ou d'une relation père/fils (Captains Courageous, Boys Town, etc.) et se déroulent souvent dans des univers homosociaux exclusivement masculins (le bateau de pêche de Captains Courageous, la ville de garçons de Boys Town, l'atelier d'Edison, the Man, le régiment de Rangers de Northwest Passage, etc.). De même, un article publié par le magazine Motion Picture explique que Tracy est plus à l'aise avec les hommes qu'avec les femmes ("[Spencer is] better with men»), comme en témoigne par exemple le fait qu'il se transforme en véritable moulin à paroles lorsqu'il mange à la cafétéria de la MGM en compagnie de Clark Gable et Robert Taylor à une table « réservée aux hommes (For Men Only) ${ }^{17}$ ». Comme je l'ai rappelé plus haut, un certain nombre d'hommes supportèrent difficilement la dépendance économique vis-à-vis des femmes et l'assignation à une sphère domestique traditionnellement perçue comme féminine qui découlait de leur chômage forcé. On peut ainsi supposer que le succès de Tracy dans des films centrés sur des amitiés viriles et des communautés exclusivement masculines s'enracine dans un fantasme de fuite loin des femmes et du féminin.

La persona de l'acteur s'inscrit en cela dans une tendance dominante du paysage idéologique de la seconde moitié des années 1930. Hollywood se tourne notamment vers le western ou le film d'aventure, deux « genres masculins ${ }^{18}$ » qui connaissent alors un regain de popularité (Dhommée 2002 : 276-278), et de nombreux romans donnent également corps à ce "fantasme masculin d'évasion " que Michael Kimmel ([1996] 2012 : 154-155) identifie par exemple dans la nouvelle de James Thurber The Secret Life of Walter Mitty, publiée en 1939 dans le New Yorker, ou encore dans les récits de Raymond Chandler dont les détectives « durs à cuire » évoluent dans une " jungle urbaine » aussi dangereuse que l'Ouest des cow-boys ou que l'Afrique de Tarzan. De son côté, Tracy éprouve sa masculinité au sein d'une nature sauvage dans des films comme Captains Courageous, Stanley and Livingstone ou Northwest Passage.

On peut s'étonner que The Snows of Kilimanjaro (1936) d'Ernest Hemingway, une nouvelle emblématique de cette projection fantasmatique de la masculinité virile dans un univers sauvage loin du foyer, ait été publiée à l'origine dans le magazine Esquire qui destine par ailleurs à son lectorat masculin des articles sur la cuisine, la mode ou la décoration d'intérieur (Breazeale 1994: 6). Cette association paradoxale permet cependant de saisir le lien entre la popularité des fictions d'évasion masculine et l'hégémonie d'un nouveau modèle de masculinité qui permet notamment aux hommes 
d'investir un univers jusqu'ici perçu comme féminin, celui de la consommation. Comme les photos de pin-up qui ornent les pages du magazine (Breazeale 1994: 9-10), la prose viriliste d'Hemingway offre en effet la garantie qu'Esquire « n'est pas pour les "tapettes (sissies)" » (Pendergast 2000 : 212). Plus largement, l'enjeu est ici d'exorciser la menace de féminisation qui plane sur l'homme urbain et moderne qui consomme et accorde une attention particulière à son apparence. Si la création d'Esquire en 1933 confirme la fortune de cette "masculinité moderne » (Pendergast $2000:$ 206-223), cette dernière commence à s'imposer sur les pages des magazines américains au tournant des années 1920 (Pendergast 1997: 55-80), et s'enracine plus profondément dans la modernisation et l'urbanisation accrues des États-Unis depuis la fin du XIX ${ }^{e}$ siècle, qui s'accompagnent entre autres de la bureaucratisation de la sphère professionnelle et de l'émergence de la société de consommation (Kimmel [1996] 2012 : 61-136).

L'image des stars hollywoodiennes masculines des années 1910-1920 exprime d'ailleurs les contradictions idéologiques qui accompagnent cette reconfiguration des normes de masculinité. Tandis que Rudolph Valentino est pour beaucoup l'incarnation par excellence de l'efféminement de l'homme américain moderne (Hansen 1991: 245-268, Studlar 1996: 150-198), Douglas Fairbanks en constitue en quelque sorte l'antidote (Studlar 1996: 10-89). Dans The Mollycoddle (Victor Fleming, 1920), il incarne par exemple un homme urbain et sophistiqué qui renoue avec son américanité et sa masculinité en rejouant la conquête de l'Ouest, et réhabilite ainsi une virilité américaine ancestrale menacée par les « excès de la civilisation (overcivilization) ». C'est dans cet héritage que s'inscrivent des films comme Captains Courageous et Northwest Passage où Tracy initie à la virilité un jeune homme (incarné respectivement par Freddie Bartholomew et Robert Young) que son éducation a tenu à l'écart d'une nature sauvage au contact de laquelle il n'a donc pas pu aiguiser sa masculinité. Si la prédilection de Tracy pour les amitiés viriles et les aventures loin du foyer est liée au contexte idéologique de la Dépression, elle s'appuie donc également sur une tradition plus ancienne de discours et de représentations qui se développent en réaction à ce qui est perçu comme une dévirilisation de l'homme américain à l'ère de la modernité et de la consommation.

L'idée selon laquelle la masculinité de Tracy serait « naturelle » est centrale à sa persona et se décline principalement de deux manières. Comme je l'ai souligné plus haut, les personnages incarnés par la star évoluent souvent dans un univers naturel loin du foyer et de la ville moderne. Dans le même esprit, les photos de l'acteur publiées dans la presse le montrent souvent dans un décor naturel, en train de camper avec ses amis, de revenir de la chasse, de labourer un champ et de s'occuper de ses chevaux dans son ranch, etc. ${ }^{19}$. À cette iconographie qui construit Tracy comme un homme dont la masculinité s'épanouit au contact de la nature, s'ajoute un ensemble de discours qui insistent sur le "naturel» de l'acteur. La "spontanéité » de son jeu est souvent soulignée par la presse et louée par ses fans. Dès 1933, dans un article de Photoplay qui interroge Fay Wray sur la manière dont ses partenaires masculins abordent les scènes d'amour, l'actrice déclare à propos de Tracy : «il est si spontanément naturel que l'on oublie qu'il est en train de jouer ${ }^{20} »$. En 1938, une lectrice du même magazine écrira à son sujet « qu'il ne donne pas l'impression de jouer, mais plutôt qu'il est Spencer luimême $^{21}$ ». Un journaliste, présenté par Screenland comme un ami et confident de la star, explique que si Tracy parvient à atteindre cet idéal de naturel que poursuivent tous les acteurs hollywoodiens, c'est parce qu'il « est l'une des personnes les plus naturelles et sincères [qui existent] ${ }^{22}$ ». Les déclarations de la star sur la manière dont il aborde son 
travail d'acteur renforcent cette image d'homme «vrai » et « authentique » qui n'est jamais dans la " $\operatorname{pose}^{23}$ ", aussi bien dans sa vie que devant la caméra. Après avoir expliqué à Gladys Hall qu'il n'a pas conscience des techniques qu'il utilise en tant qu'acteur, il abonde dans le sens de la journaliste lorsque celle-ci affirme qu'il fait partie des « instinctifs ${ }^{24} »$.

Conformément à la reconfiguration des normes de masculinité qui s'est opérée au tournant du $\mathrm{xx}^{\mathrm{e}}$ siècle, la persona de Tracy se caractérise donc par une prééminence du " motif de la nature », conçue à la fois comme un espace revitalisant pour les hommes et comme une essence masculine profonde (Courcoux $2017: 70$ ). Chez des stars comme James Cagney ou Clark Gable, cette masculinité soi-disant essentielle s'exprime principalement à travers un tempérament violent et impulsif. Après avoir marqué les esprits dans des rôles d'hommes brutaux ${ }^{25}$, Gable déclare par exemple dans Modern Screen qu'il a " toujours vécu de manière impulsive ${ }^{26}$ ». Tracy se rapproche par certains aspects de ce modèle de masculinité axé entre autres sur les idées de force physique et d'agressivité, mais il n'a toutefois pas une réputation d'«homme des cavernes (caveman) ${ }^{27}$ » comparable à celle de Cagney ou Gable, notamment parce que d'autres aspects de sa persona le ramènent plus du côté de la civilisation, de la modération et du féminin, comme je le montrerai plus bas. Cependant, même si sa masculinité n'est pas vraiment perçue comme "naturelle » en tant qu'expression d'une essence primitive, violente ou pulsionnelle, les discours sur son jeu d'acteur indiquent que son image d'homme "authentique», "spontané» et «instinctif» le rattache à une telle conception essentialiste de la masculinité et tend à l'éloigner d'une masculinité «trop civilisée (overcivilized) » placée sous le signe de l'artificialité, de la sophistication et de la performance.

Cet aspect de sa persona se nourrit notamment d'un ensemble de représentations et de discours qui soulignent le peu d'intérêt que Tracy manifeste pour la consommation et le travail de l'apparence, malgré la place centrale de ces pratiques dans la définition de la « masculinité moderne » qui assoit alors son hégémonie culturelle (Pendergast 2000 : 206). Libeled Lady (Jack Conway, 1936) lui donne le rôle d'un journaliste un peu rustre, opposé à l'élégant William Powell qui parvient à s'introduire dans une famille de la haute société grâce à sa maîtrise des codes vestimentaires et comportementaux de la classe supérieure. Face à la «masculinité instable» de Powell, qui tend vers le « féminin » du fait de son recours à la « mascarade » et à la «tromperie » (Glitre 2006: 79), Tracy incarne une masculinité plus monolithique et stéréotypée, placée dès les premières scènes dans un rapport antagoniste aux femmes et au féminin à travers ses confrontations avec Jean Harlow au sujet de leur mariage. Tandis que Powell multiplie les tenues sophistiquées tout au long du film, Tracy commence par être ridiculisé dans son costume de cérémonie, dont il se débarrasse à la première occasion. Dans le même esprit, une lectrice de Photoplay qualifie Tracy d'homme « rustique » auquel « un nœud papillon et une queue-de-pie n'iraient pas très bien ${ }^{28}$ ». Un journaliste de Modern Screen raconte que l'acteur, gêné par un costume élégant qu'il devait porter pour une scène du film Mannequin (Frank Borzage, 1937), s'empressait d'aller se changer à chaque pause ${ }^{29}$, tandis que son épouse confirme la prédilection de Tracy pour la simplicité vestimentaire en expliquant qu'il «se contente d'une vieille veste et d'un vieux pantalon, qu'ils soient assortis ou non ${ }^{30} »$. Alors que l'identité masculine tend de plus en plus à apparaître comme une construction sociale depuis le tournant du $\mathrm{xx}^{\mathrm{e}}$ siècle (Courcoux 2017 : 92) et que la «masculinité moderne » privilégie «l'apparence » à la 
« substance » (Pendergast $1997: 64-65 ; 2000: 220-223)$, il est probable que le mépris de Tracy pour la consommation et la sophistication vestimentaire cultive son image d'homme "authentique » dont le genre ne relève pas d'une performance mais d'une essence profonde, et contribue ainsi à en faire un antidote à la menace de dénaturalisation qui plane alors sur la masculinité.

Dans une scène de Boom Town qui s'appuie sur cet aspect de son image, Gable le force à étoffer sa garde-robe. Le comique réside ici dans l'inadéquation entre la masculinité « rustique » de la star et un souci de l'apparence associé aux femmes et aux « hommes modernes ", ainsi que dans l'exaspération de Tracy qui doit subir les attouchements d'un tailleur français connoté comme homosexuel. En gardant ses distances avec le nouveau modèle de "masculinité moderne », Tracy se prémunit donc également contre toute association à la féminité et à l'homosexualité. Cela est d'autant plus nécessaire que son image d'homme " plus à l'aise avec les hommes " est entretenue non seulement par les magazines, mais aussi par ses rôles à l'écran, notamment dans ses films avec Gable (San Francisco, Test Pilot et Boom Town) où il occupe de manière récurrente la "place de la femme» au sein du couple homosocial formé par les deux stars masculines. Dans Test Pilot, Gable lui demande ainsi de l'accompagner au rayon lingerie d'un grand magasin pour l'aider à choisir la chemise de nuit qu'il compte offrir à Myrna Loy, et essaie le vêtement féminin sur un Tracy embarrassé. Une photo de la scène figure d'ailleurs sur certaines affiches, avec un commentaire qui explique: "Clark se sert de Spencer pour remplacer Myrna! Spencer veut bien mais ne peut pas... si vous voyez ce que je veux dire ${ }^{31}$. » Même si la gêne manifestée par Tracy exorcise dans une certaine mesure les connotations féminines et homosexuelles que cette scène pourrait conférer à sa persona, sa proximité avec Loy pendant tout le film, dont il est un confident privilégié et avec laquelle il partage une même " position féminine » vis-à-vis de Gable (Dhommée 2002 : 296), indique que la masculinité hégémonique incarnée par Tracy ne se construit pas uniquement en opposition aux femmes et au féminin.

\section{Un père doux et compréhensif}

21 Malgré sa prédilection pour des univers virils dont les femmes sont exclues, Tracy parvient à s'attirer les faveurs des spectatrices au point d'être l'acteur le plus apprécié du public féminin au tournant des années 1940 (Gallup 1979), un exploit d'autant plus étonnant qu'il n'a pas l'image d'un séducteur, contrairement à la majorité des stars masculines plus populaires auprès des femmes. Lorsqu'il est opposé à Clark Gable, c'est toujours ce dernier qui séduit le personnage féminin tandis que Tracy tient le rôle de l'ami fidèle qui reste célibataire. Dans Mannequin et I Take This Woman (W. S. Van Dyke, 1940), les hérö̈nes incarnées par Joan Crawford et Hedy Lamarr sont passionnément amoureuses d'un autre homme et apprécient avant tout chez Tracy sa bonté d'âme. En effet, contrairement à des acteurs comme Robert Taylor ou Tyrone Power, il n'est pas considéré comme beau. Une fan écrit ainsi que les gros plans de son visage n'ont pas pour but d'être des «jolies images" mais de mettre en évidence «ses yeux tendres, tellement transparents que l'on peut voir son âme ${ }^{32}$ ». Dans le même courrier, elle le qualifie de "gentil et sentimental » et loue l'empathie dont il fait preuve en tant qu'acteur $^{33}$. Lorsqu'elle essaie d'expliquer son succès exceptionnel auprès des spectatrices, une journaliste de Motion Picture explique également qu'il «incarne l'homme gentil, tendre et compréhensif », avant de mentionner sa «fidélité totale » et 
son « dévouement indéfectible » comme des qualités particulièrement attractives pour les femmes au même titre que son côté protecteur ${ }^{34}$. Cette image est cultivée par la plupart de ses rôles à l'écran, notamment lorsqu'il joue au côté de Gable. Alors que ce dernier incarne des personnages égocentriques qui font inévitablement souffrir les femmes, Tracy apparaît au contraire comme un homme qui respecte, écoute et comprend les femmes.

De surcroît, même si ses personnages s'accomplissent le plus souvent dans la sphère professionnelle et/ou dans la confrontation avec une nature sauvage, sa masculinité ne se construit pas cependant dans un antagonisme avec la sphère domestique. Cet aspect de son image est sensible dans des films comme Mannequin ou Boom Town, mais aussi et surtout dans son premier film avec Katharine Hepburn, Woman of the Year (George Stevens, 1942), dans lequel il tente de sauver un foyer menacé par l'ambition de son épouse féministe, à qui il finira par faire prendre conscience de la valeur du mariage. Dans le même esprit, un article de Motion Picture explique ainsi qu'avant d'être un acteur, Tracy est surtout un mari et un père ${ }^{35}$, tandis que la star répète en interview qu'il doit tout à sa femme et que son fils est la chose la plus importante de sa vie ${ }^{36}$. En réaction à la baisse de la natalité et du taux de mariage causée par la Dépression (Dhommée 2002: 251-253, Olszowka 2014: 168), Hollywood revalorise alors les institutions conjugale et familiale ${ }^{37}$, et la persona de Tracy contribue à cette tendance idéologique. Le fait que son couple ait surmonté des épreuves difficiles est rappelé dans de nombreux articles et constitue sans doute une inspiration pour de nombreuses familles américaines fragilisées par la crise. Pendant la seconde moitié de la décennie, les fan magazines publient de nombreuses photos de l'acteur en compagnie de son épouse ou de ses enfants ${ }^{38}$, une iconographie qui contribue à le construire comme un homme épanoui dans ses rôles de père et de mari. Ajoutée à son image de "gentleman ${ }^{39}$ » respectueux des femmes, cette intégration au foyer le place aux antipodes des figures de gangster qui permirent notamment à Edward G. Robinson, James Cagney et Paul Muni de triompher au box-office pendant les années les plus noires de la Dépression. Alors que les héros de Little Caesar (Mervyn LeRoy, 1931), The Public Enemy (William A. Wellman, 1931) et Scarface (Howard Hawks, 1932) se montrent particulièrement violents envers les femmes et rejettent le féminin sous toutes ses formes (Sandeau 2015 : 180-183), Tracy incarne à la ville comme à l'écran une masculinité américaine qui s'est réconciliée avec les femmes et ce lieu féminin par excellence qu'est le foyer.

De surcroît, la persona de l'acteur témoigne d'une redéfinition des normes de masculinité qui repose en partie sur l'assimilation de qualités et de valeurs associées au féminin. En effet, la perturbation de l'ordre de genre provoquée par la Dépression n'a pas seulement résidé dans une reconfiguration des rôles et rapports de genre au sein de nombreux couples, mais également dans le fait que des "valeurs masculines» qui étaient glorifiées pendant les années 1920 (business, agressivité, compétition, etc.) ont alors perdu un peu de leur hégémonie (McElvaine [1984] 2009 : 196-223). Pour beaucoup d'Américainees, c'est l'individualisme compétitif qui a plongé le pays dans la crise, et ce sont des valeurs culturellement associées au féminin qui vont l'en sortir: la coopération, l'altruisme, l'empathie, etc. (Hanson 2008 : 113 ; Sandeau 2015 : 175-176). La déstabilisation provisoire du capitalisme patriarcal suite au Krach de 1929 touche donc à la fois un système économique et l'idéal masculin qui lui est lié : le self-mademan, alors conçu comme un homme viril et déterminé qui s'est fait tout seul à la force du poing et évolue dans la jungle de la concurrence sauvage (Sandeau 2015 : 175-176). 
Profondément ambivalents dans leur manière de glorifier cette figure tout en la condamnant, les films de gangsters cités plus haut sont emblématiques des doutes dont le self-made-man fait l'objet durant la Dépression (178-192). Avec le retour à la prospérité, ce modèle masculin est progressivement revalorisé, notamment grâce à une mise à distance de sa version outrancièrement agressive personnifiée par les gangsters du début de la décennie, qui étaient déjà fustigés en tant qu'incarnations déviantes et pathologiques de cet idéal masculin.

Les films dans lesquels Tracy incarne un self-made-man témoignent de la revalorisation/redéfinition de cette figure au cours de la seconde moitié de la décennie. Dans Edison, the Man, il n'a pas pour but l'enrichissement personnel ou l'ascension sociale, mais œuvre pour le bien commun en travaillant sur des inventions qui amélioreront la condition de vie des gens ordinaires. De son côté, Mannequin enracine les ambitions excessives du self-made-man personnifié par Tracy dans une masculinité que l'épouse dévouée incarnée par Joan Crawford se donne pour mission de réguler. Lorsqu'elle lui rappelle que les bulles de savon les plus petites sont les plus solides tandis que les plus grosses sont plus fragiles et risquent à tout moment d'éclater, il n'a aucune peine à saisir le sens de cette métaphore («La morale doit être : "Ne monte pas trop haut" 40 "), mais ajoute immédiatement: "C'est vrai pour les bulles de savon féminines, mais il y a quelque chose chez l'homme qui le pousse à aller plus loin, à essayer de voir jusqu'où il peut grossir sans éclater ${ }^{41}$. » Quand il fera faillite à la fin du film, son épouse l'encouragera à reconstruire ce qu'il a perdu («Ce que tu as fait, tu peux le refaire »), tout en lui expliquant qu'elle restera à ses côtés pour s'assurer qu'il " ne grossisse pas au point d'éclater ${ }^{42}$ ». À travers cette métaphore des bulles de savon, le film défend un capitalisme patriarcal raisonnable, que Tracy pourra personnifier s'il fait sienne la sagesse féminine de Crawford pour devenir en quelque sorte la «bulle de savon féminine " contre laquelle il définissait sa masculinité un peu plus tôt. Cette conclusion illustre bien la complexité de l'opération idéologique dont la persona de Tracy est le lieu, puisque tout en reconnaissant la nécessité de "féminiser " la masculinité hégémonique américaine, le film réaffirme en même temps l'ordre de genre et la viabilité du système capitaliste. En contradiction avec son image de selfmade-woman à l'ambition insatiable, Crawford finit en effet par prendre conscience que son «travail» est de se dévouer à son mari pour que celui-ci puisse réussir professionnellement, une trajectoire qui la rapproche de l'épouse de Tracy telle que construite par la presse pendant la période ${ }^{43}$. Corrélativement, le personnage incarné par Tracy n'est jamais véritablement dévalorisé en tant que self-made-man capitaliste, signe que le film cherche moins à déconstruire cet idéal qu'à le réhabiliter en le réformant. Dépeint comme un patron exemplaire qui traite ses employés comme ses égaux, il n'est pas présenté comme le responsable de la faillite de son entreprise, et la dernière scène laisse entendre qu'il renaîtra de ses cendres, à la manière des héros de Boom Town qui ne cessent de prouver leur capacité à « réussir à partir de rien ».

Troisième film centré sur une amitié virile entre Tracy et Gable, Boom Town permet de saisir les convergences et divergences dans l'image des deux stars. Si les prospecteurs de pétrole qu'ils incarnent sont tous deux des self-made-men dont la persévérance et le goût du risque sont glorifiés tout au long du film, la sagesse de "Square John » (Tracy) contraste avec l'immaturité de «Big John" (Gable). Le film reprend ici la dynamique qui avait fait le succès de leurs collaborations précédentes - dans San Francisco et Test Pilot. Gable et Tracy sont à chaque fois opposés en tant qu'incarnations de deux facettes complémentaires de la masculinité américaine. Tandis que Gable est construit comme 
un descendant des pionniers qui personnifie à la fois l'esprit de conquête, l'opportunisme et l'individualisme compétitif, Tracy incarne plutôt les valeurs d'entraide, de solidarité, mais aussi les institutions telles que la loi ou la famille. Son rôle consiste simultanément à civiliser la force brute, quasi pulsionnelle, incarnée par Gable et à reconnaître en même temps l'importance de cette force pour la société américaine. Sa fonction dans la légitimation de la "masculinité excessive » de son partenaire est illustrée par le plaidoyer qu'il prononce à la fin de Boom Town pour défendre Gable face à un tribunal qui l'accuse d'avoir violé la loi anti-trust :

McMasters est un entrepreneur. Si les voitures n'existaient pas, il conduirait un chariot. Ça a toujours été les gens de son espèce qui ont ouvert le pays et qui en ont fait ce qu'il est. Je pose donc la question : cela va-t-il devenir interdit, aux ÉtatsUnis, pour un homme comme lui, de gagner un million de dollars avec son cerveau ou avec ses mains ? Parce que si c'est le cas, alors on ferait mieux d'arrêter de parler de cette terre-de-toutes-les-opportunités ${ }^{44}$.

La persona de Spencer Tracy se révèle ainsi profondément ambivalente puisque, tout en incarnant comme Gable une masculinité virile, un self-made-man déterminé et agressif, il est présenté comme moralement supérieur à son comparse, face auquel il personnifie un modèle de sagesse, de bonté et de maturité. Replacée dans le contexte de la seconde moitié des années 1930, l'image de Tracy semble donc avoir pour fonction principale de "moraliser» le capitalisme et l'individualisme sauvages que la crise de 1929 a momentanément délégitimé aux yeux de beaucoup d'Américain·es. Plus exactement, on peut soutenir, en reprenant les termes de Richard Dyer (2004a : 21), que l'image de Tracy résout «magiquement " la "contradiction idéologique » entre, d'une part, les valeurs masculines du capitalisme débridé qui retrouvent leur hégémonie avec le retour à la prospérité, et d'autre part, les valeurs féminines qui sont alors perçues par beaucoup comme un antidote à la crise touchant le pays.

Ce versant "féminin " de la persona de Tracy est particulièrement sensible dans les films qui le construisent comme un homme humble et généreux qui consacre sa vie à aider les plus démuni-e-s. Dans I Take This Woman, il incarne ainsi un docteur qui préfère vivre modestement dans une clinique dont la clientèle est pauvre et immigrée, plutôt que de faire carrière dans un hôpital prestigieux dont les patients appartiennent à la haute société et sont majoritairement hypocondriaques. Ses rôles de prêtre contribuent de manière décisive à la cristallisation de cette image d'homme altruiste : le Père Mullin de San Francisco, et surtout le Père Flanagan de Boys Town et Men of Boys Town (Norman Taurog, 1941), deux films qui s'inspirent de l'œuvre d'un prêtre catholique connu pour avoir fondé un village accueillant les jeunes garçons délinquants ou abandonnés. Dans un article publié par Photoplay en 1938, Tracy explique que même s'il est un "bon businessman ", il n'est pas pour autant "dévoré par l'ambition de gagner beaucoup d'argent", et projette plutôt de mettre sa notoriété d'acteur au service de causes comme la lutte contre le cancer ou la délinquance juvénile ${ }^{45}$. Quelques semaines après la sortie de Boys Town, une journaliste de Modern Screen explique que le secret de sa grandeur est sa générosité, le fait qu'il se préoccupe sincèrement des autres personnes, qu'il soit quelqu'un d'attentionné (caring) ${ }^{46}$. Dans un contexte idéologique où l'idéal de l'homme américain agressif et individualiste perd un peu de son hégémonie, Tracy incarne une reconfiguration des normes de masculinité qui intègre des qualités alors considérées comme féminines : modération, altruisme, care, tendresse ${ }^{47}$, compassion ${ }^{48}$, etc. 
La centralité des relations masculines et la marginalisation corrélative des femmes dans la plupart des films tournés par Tracy à l'époque, indiquent que cette appropriation par les hommes de traits culturellement associés au féminin consiste en grande partie en une dépossession. Les films dans lesquels Tracy est sauvé par une femme, comme Fury ou Mannequin, sont relativement rares. Généralement, c'est lui qui aide un personnage masculin immature - souvent incarné par Clark Gable ou Mickey Rooney - à accéder à une masculinité moins égoïste et plus modérée. Les discours des fan magazines sur les épreuves traversées par Tracy évoluent d'ailleurs significativement pendant la période. Après avoir insisté sur le rôle décisif joué par son épouse dans la renaissance de la star suite à ses égarements du milieu de la décennie (relation avec Loretta Young, départ du foyer, alcoolisme) ${ }^{49}$, la presse tend plutôt à présenter Tracy comme un homme qui a trouvé en lui les ressources pour se régénérer tel un « Phoenix » et peut maintenant jouer un rôle de modèle et de guide vis-à-vis des autres hommes ${ }^{50}$.

C'est avant tout en incarnant des figures paternelles que Tracy va contribuer à cette appropriation/dépossession par les hommes de qualités conventionnellement attribuées aux femmes. La reconfiguration de la masculinité dans le contexte de la Dépression passe par une mise en avant de la paternité qui s'explique entre autres par la nécessité pour beaucoup d'Américains de redéfinir leur identité d'homme en dehors de la sphère professionnelle, et donc notamment au sein du foyer (Olszowka $2014: 167$ ). On peut également voir la prolifération de «figures paternelles traditionnelles » dans les médias de l'époque (188-190) comme une réponse aux doutes que la Dépression a fait planer sur le patriarcat pendant la première moitié de la décennie. Aux antipodes des pères défaillants qu'incarnaient par exemple Wallace Beery dans The Champ ou Lionel Barrymore dans A Free Soul (Clarence Brown, 1931), les figures paternelles personnifiées par Tracy sont solides et exemplaires. S'il faut mentionner en premier lieu les rôles de père de substitution qu'il joue vis-à-vis de Freddie Bartholomew (Captains Courageous) et Mickey Rooney (Boys Town, Men of Boys Town), l'inspiration qu'il constitue pour Robert Young dans Northwest Passage ou Clark Gable dans San Francisco nourrit également cet aspect de son image ${ }^{51}$. Premier grand succès de Tracy dans un rôle de père, Captains Courageous témoigne tout particulièrement de l'ambivalence idéologique de cette figure dans le contexte de la seconde moitié de la décennie. En faisant de Tracy un homme du peuple qui parvient à remettre sur le droit chemin l'enfant capricieux d'un riche industriel (Melvyn Douglas), le film met l'accent sur le paramètre de classe, tout en évacuant la question des rapports de genre. Contrairement à She Married Her Boss (Gregory La Cava, 1935) avec lequel il possède un certain nombre de points communs, Captains Courageous ne donne aucun rôle clef à un personnage féminin dans la réforme de la famille de classe supérieure qu'il met en scène. Tracy remplace à la fois le père trop permissif incarné par Douglas et la mère dont le film souligne d'emblée l'absence. Cet homme "dont la force brute cache une profonde douceur ${ }^{52}$ » apprend ainsi au jeune garçon à se soumettre à l'autorité et à se viriliser en même temps qu'il lui offre l'amour et la tendresse qui lui manquent. À travers ce personnage de "père » idéal, Tracy incarne donc une masculinité autosuffisante qui peut se passer des femmes parce qu'elle cumule qualités «masculines» et «féminines ». 


\section{Entre masculinity et manhood}

Pour conclure, je ferai l'hypothèse que la persona de Tracy témoigne de la cohabitation, pendant la période, entre deux conceptions de la masculinité qui sont généralement distinguées en anglais par les termes de manhood (ou de manliness) d'un côté, et de masculinity de l'autre. En s'appuyant notamment sur les travaux de Gail Bederman (1995), Michael Kimmel ([1996] 2012) et E. Anthony Rotundo (1993), Charles-Antoine Courcoux (2017 : 64-115) a souligné les modalités et enjeux de la reconfiguration de la masculinité hégémonique américaine qui s'est opérée au tournant $d u x^{e}$ siècle. Prisée par les Américains de classe moyenne tout au long du XIXe et jusque dans les premières décennies $\mathrm{du} \mathrm{xx}^{\mathrm{e}}$ siècle, la manhood (ou manliness) est conçue comme une "capacité à contrôler ses pulsions et ses désirs, à se civiliser en accord avec les qualités morales dont la femme est désormais dépositaire » (66). Pendant cette période, l'adjectif "manly» connote ainsi "autorité morale ", "probité ", «force de caractère » et "maîtrise de soi ", un ensemble de qualités à travers lesquelles les hommes blancs de classe moyenne cherchent alors à légitimer leur suprématie, en alimentant un discours sur la "civilisation" justifiant à la fois leurs privilèges de race et de genre (65). "Qualité intérieure " possédée par l'homme " autonome et responsable », la manhood est pensée comme l'opposé de la childhood, c'est-à-dire de l'« infantilité », tandis que la masculinity, qui s'impose comme la nouvelle norme dominante de masculinité au tournant du $\mathrm{xx}^{\mathrm{e}}$ siècle, se construit plutôt en opposition à la feminity (67). « Articulée autour de la force musculaire, de l'instinct, d'une forme de bestialité et du déni de la féminité », la masculinity s'écarte progressivement de l'idéal de "modération» et d'«accomplissement vertueux» que constitue la manhood, pour enraciner la masculinité dans une nature sauvage opposée à la civilisation et au féminin (66-67).

Comme Douglas Fairbanks pendant les années 1910-1920, Clark Gable peut être tenu comme l'incarnation emblématique de cette masculinity dans le paysage hollywoodien des années 1930. Tout en s'y apparentant par de nombreux aspects (prédilection pour les espaces naturels revitalisants et les univers homosociaux masculins, corps trapu et vigoureux, tempérament agressif, etc.), Tracy s'en écarte également d'une manière qui le rapproche de la manhood. Si sa première apparition dans San Francisco insiste sur sa supériorité physique lorsqu'il terrasse Gable sur un ring de boxe, son corps n'est pourtant pas exhibé comme celui de son partenaire. Placé du côté de la spiritualité et de la raison, son personnage de prêtre catholique est en effet opposé au personnage impulsif incarné par Gable, " force de la nature ${ }^{53}$ » que Tracy tente de mettre au service du bien, selon une dynamique que l'on retrouve à la même période chez le duo James Cagney/Pat O'Brien dans Angels with Dirty Faces (Michael Curtiz, 1938). Ce rôle civilisateur, qui le rapproche des personnages féminins incarnés par Jeannette MacDonald, Myrna Loy ou Claudette Colbert, indique que sa masculinité s'oppose donc moins à la féminité qu'à la childhood, comme en témoignent ses nombreux rôles de pères symboliques qui guident des enfants vers l'âge adulte: Freddie Bartholomew dans Captains Courageous, Mickey Rooney dans Boys Town et Men of Boys Town, mais également Gable, dans la mesure où ce dernier est souvent caractérisé comme un homme immature qui doit apprendre à grandir.

31 La persona de Tracy semble donc témoigner d'une résurgence momentanée de traits associés à la manhood pendant les années 1930, à l'encontre de la prédominance croissante de la masculinity dans la définition de la masculinité hégémonique 
américaine au cours des premières décennies $d u x^{e}$ siècle. En suscitant de la méfiance envers un «individualisme sauvage (rugged individualism)» perçu comme masculin (Hanson 2008 : 114), la Dépression fut probablement la cause de la revalorisation d'un idéal de masculinité plus modérée et responsable. Loin des excès de la masculinity sauvage et débridée qui a plongé le pays dans la crise, Tracy incarne cette nouvelle manhood qui remettra les États-Unis dans le droit chemin. Plus exactement, l'acteur réalise la synthèse de ces deux conceptions de la masculinité américaine. Agressif, énergique et robuste, il constitue en même temps un modèle de sagesse, de maturité et de moralité. De même, malgré sa prédilection pour des univers homosociaux, virils et violents, il s'accommode aussi très bien des femmes et du féminin. Cette ambivalence de la persona de Tracy permet ainsi de comprendre comment il a pu devenir aussi populaire aux États-Unis pendant la période, et témoigne de la plasticité de la masculinité hégémonique américaine, dont la capacité d'adaptation est particulièrement manifeste lorsque l'ordre de genre est perturbé, dans des contextes tels que la Dépression.

\section{BIBLIOGRAPHIE}

Armengol, Josep M., 2014, « Gendering the Great Depression: Rethinking the Male Body in 1930s American Culture and Literature ", Journal of Gender Studies, vol. 23, n 1, p. 59-68.

Balio, Tino, 1995, Grand Design: Hollywood as a Modern Business Enterprise 1930-1939, Berkeley, Los Angeles, Londres, University of California Press.

Baxandall, Rosalyn, Gordon, Linda et Reverby, Susan (dir.), [1976] 1995, America's Working Women: A Documentary History, 1600 to the Present, New York, Londres, W. W. Norton \& Company.

Becker, Christine, 2011, «Clark Gable: The King of Hollywood », dans A. L. McLean (dir.), Glamour in a Golden Age: Movie Stars of the 1930s, New Brunswick, Rutgers University Press, p. 245-266.

Bederman, Gail, 1995, Manliness and Civilization: A Cultural History of Gender and Race in the United States, 1880-1917, Chicago, Londres, The University of Chicago Press.

Bergman, Andrew, 1971, We're the Money: Depression America and its Films, New York, New York University Press.

Bourget, Jean-Loup, 1983, Le Cinéma américain 1895-1980 : De Griffith à Cimino, Paris, PUF.

Breazeale, Kenon, 1994, «In Spite of Women: Esquire Magazine and the Construction of the Male Consumer », Signs, vol. 20, nº 1, p. 1-22.

Cervulle, Maxime, 2013, Dans le blanc des yeux. Diversité, racisme et médias, Paris, Éditions Amsterdam.

Connell, Raewyn, 2014, Masculinités : Enjeux sociaux de l'hégémonie, Paris, Éditions Amsterdam.

Connelly, Timothy, 2004, « He Is As He Is - and Always Will Be: Clark Gable and the Reassertion of Hegemonic Masculinity ", dans P. Powrie, A. Davis et B. Babington (dir.), The Trouble with Men: Masculinities in European and Hollywood Cinema, Londres, New York, Wallflower Press, p. 34-41. 
Courcoux, Charles-Antoine, 2017, Des machines et des hommes. Masculinité et technologie dans le cinéma américain contemporain, Chêne-Bourg, Georg éditeur.

Curtis, James, 2011, Spencer Tracy: A Biography, Londres, Hutchinson.

Dhommée Isabelle, 2002, Les Cinq « empoisonneuses » : G. Garbo, J. Crawford, M. Dietrich, M. West, $K$. Hepburn et les États-Unis des années trente : analyse du phénomène social de la star, Villeneuve d'Ascq, Presses universitaires du Septentrion.

Doss, Erika, 1997, « Toward an Iconography of American Labor: Work, Workers, and the Work Ethic in American Art, 1930-1945 », Design Issues, vol. 13, nº 1, p. 53-66.

Dyer, Richard, [1979] 2004a, Le star-système hollywoodien, Paris, L'Harmattan.

Dyer, Richard, [1986] 2004b, Heavenly Bodies: Film Stars and Society, Londres, New York, Routledge. Dyer, Richard, 1997, White, Londres, New York, Routledge.

Filene, Peter G., [1974] 1998, Him/Her/Self: Gender Identities in Modern America, Baltimore, Londres, Johns Hopkins University Press.

Gallup, George, 1979, Gallup Looks at the Movies: Audience Research Reports 1940-1950, Wilmington, Delaware, Scholarly Resources.

Glitre, Kathrina, 2006, Hollywood Romantic Comedy: States of the Union, 1934-65, Manchester, New York, Manchester University Press.

Guillaumin, Colette, 1992, Sexe, race et pratique du pouvoir. L'idée de nature, Paris, Indigo, Côtéfemmes éditions.

Handel, Leo A., 1950, Hollywood Looks at Its Audience: A Report of Film Audience Research, Urbana, University of Illinois Press.

Hansen, Miriam, 1991, Babel and Babylon: Spectatorship in American Silent Film, Cambridge, Londres, Harvard University Press.

Hanson, Philip, 2008, This Side of Despair: How the Movies and American Life Intersected during the Great Depression, Madison, Fairleigh Dickinson University Press.

Kimmel, Michael, [1996] 2012, Manhood in America: A Cultural History, New York, Oxford, Oxford University Press.

Komarovsky, Mirra, [1940] 1971, The Unemployed Man and His Family: The Effect of Unemployment upon the Status of the Man in Fifty-Nine Families, Oxford, AltaMira Press.

Loew, Brenda (dir.), 2008, Spencer Tracy: Fox Film Actor: the Pre-code Legacy of a Hollywood Legend, Newton, Massachusetts, New England Vintage Film Society, Inc.

McElvaine, Robert S., [1984] 2009, The Great Depression, New York, Three Rivers Press.

Melosh, Barbara, 1993, « Manly Work: Public Art and Masculinity in Depression America », dans B. Melosh (dir.), Gender and American History Since 1890, Londres, Routledge, p. 155-181.

Mintz, Steven et Kellogg, Susan, 1988, Domestic Revolutions: A Social History of American Family Life, New York, Free Press.

Olszowka, John, Sullivan, Marnie M., Sheridan, Brian R. et Hickey, Dennis, 2014, America in the Thirties, Syracuse, NY, Syracuse University Press.

Pendergast, Tom, 1997, « “Horatio Alger Doesn't Work Here Any More”: Masculinity and American Magazines, 1919-1940», American Studies, n 38, p. 55-80. 
Pendergast, Tom, 2000, Creating the Modern Man: American Magazines and Consumer Culture, 1900-1950, Columbia, Londres, University of Missouri Press.

Penner, James, 2011, Pinks, Pansies, and Punks: The Rhetoric of Masculinity in American Literary Culture, Bloomington, Indianapolis, Indiana University Press.

Rotundo, E. Anthony, 1993, American Manhood: Transformations in Masculinity from the Revolution Era to the Modern Era, New York, Basic Books.

Sandeau, Jules, 2015, « Retour sur les films de gangsters hollywoodiens des années 1930 : codage et décodage de masculinités marginalisées ", Genre en séries : cinéma, télévision, médias, ${ }^{\circ} 1$, p. 172-195, [En ligne], http://genreenseries.weebly.com/uploads/1/1/4/4/11440046/ ges_n\%C2\%B01_sandeau.pdf, consulté le 27 novembre 2019.

Shumway, David R., 1991, « Screwball Comedies: Constructing Romance, Mystifying Marriage », Cinema Journal, vol. 30, $\mathrm{n}^{\circ}$ 4, p. 7-23.

Steinberg, Cobbett S., 1980, Film Facts, New York, Facts on File.

Studlar, Gaylyn, 1996, This Mad Masquerade: Stardom and Masculinity in the Jazz Age, New York, Columbia University Press.

Wall, Wendy L., 2009, Inventing the "American Way": The Politics of Consensus from the New Deal to the Civil Rights Movement, New York, Oxford University Press.

\section{NOTES}

1. Motion Picture, décembre 1936, p. 66.

2. Après avoir connu une période creuse pendant les années les plus dures de la Dépression, le western voit sa popularité augmenter régulièrement durant la seconde moitié de la décennie (Bergman 1971: 88, Balio 1995 : 193-195). Jean-Loup Bourget (1983 : 82) note également que nombre de productions de la période «peignent de couleurs nostalgiques ou héroïques le passé américain » et « expriment, à l'instar de la peinture des années 1930, à la fois le repli sur soi, le retour aux sources purement américaines, le sens de la communauté ».

3. Photoplay, février 1937, p. 22, 105.

4. «Beginning the absorbing biography of the fighting Irishman who battled his way through poverty and tragedy - and won » (Photoplay, février 1937, p. 23).

5. «A man of strength and courage, a man of purpose and determination, a man of talent and ability... that's Spencer Tracy » (Photoplay, octobre 1937, p. 87).

6. " "He's rugged", said Van. "He's masculine, every inch of him" [...] "He's rough and tough; he's a man » (Modern Screen, mai 1940, p. 78).

7. « turbulent Irishman » (Photoplay, avril 1937, p. 56), « fighting Irishman » (Hollywood, juillet 1937, p. 26, 59), « courageous Irishman », (Picture Play, mars 1938, p. 50), etc.

8. « Behind him generations of fighting Irish » (Photoplay, février 1937, p. 22).

9. Voir notamment l'article «That Tough Tracy Kid» (Picture Play, février 1937, p. 46-47). 
10. «He was truculent even at the beginning, causing as much trouble as possible and frightening everybody, even the doctors, out of their wits. [...] he was a husky baby born with sturdy arms » (Photoplay, février 1937, p. 22).

11. Sur ce concept, voir Maxime Cervulle (2013). Comme lui, je traduis « whiteness » par "blanchité", à la suite de Judith Ezekiel qui "s'oppose à la traduction par "blanchitude", établissant un parallèle erroné avec la notion de négritude et effaçant par là le caractère asymétrique de leurs rapports et le statut hégémonique [de la] “ whiteness" » (48).

12. Sur l'idéologie naturaliste qui s'emploie à légitimer des faits sociaux en les faisant passer pour naturels, voir notamment Colette Guillaumin (1992).

13. «He's had his difficult years, his disappointments - times when it looked like the hard knocks were avalanche. He's had his personal troubles and his professional setbacks, but neither life nor what it gave him could ever stop the force that made him take everything in his stride " (Modern Screen, septembre 1939, p. 36).

14. "Out of the ashes of his former self he has risen, as it were, a powerful Phoenix " (Motion Picture, novembre 1937, p. 86).

15. «[...] whatever causes a man to change his ways comes from somewhere within that man himself. We can't be "killed or cured" by anyone but ourselves. The conquests we achieve over ourselves come from within, or not at all. And what I want to say is this: we can achieve those conquests " (Motion Picture, novembre 1937, p. 86).

16. " "I guess it all comes down," said Spencer, "to the person who can't be discouraged, to the fellow who won't give up! No matter what odds are against him, I mean, no matter how long he has to wait and work. You can't defeat a person who has no such word as defeat in his vocabulary. Because he can't be discouraged. You can't shake a fellow loose from success if he won't give up!" " ("Good Advice from Spencer Tracy ", Screenland, juillet 1940, p. 30).

17. Motion Picture, juillet 1940, p. 27.

18. Ces genres sont «masculins" à la fois parce qu'ils mettent en scène majoritairement des hommes et parce qu'ils sont plus appréciés des hommes que des femmes (Handel $1950: 124)$.

19. Voir par exemple Photoplay, février 1937, p. 22-23; octobre 1940, p. 58-59; Screenland, novembre 1939, p. 38-39; mars 1940, p. 32-34.

20. "Spencer is the most direct screen-lover of them all, and he makes love the least like an actor. Why, he's so spontaneously natural about it that you forget he's acting at all » (Photoplay, novembre 1933, p. 52).

21. «As you watch him you have a feeling he isn't acting, that's Spencer himself, not a portrayal.» (Photoplay, juin 1938 p. 8-9).

22. "Every actor in Hollywood strives for naturalness. Because Spence is fundamentally one of the most natural and sincere people I have ever met, only naturalness and sincerity come through on the screen. " (Screenland, mai 1938, p. 64).

23. « He has no tricks [...] he doesn't pose » (Motion Picture, novembre 1937, p. 31).

24. Motion Picture, juillet 1940, p. 74.

25. Voir notamment sa prestation face à Norma Shearer dans A Free Soul (Clarence Brown, 1931 -Becker 2011: 248). Sur la virilisation de sa persona au cours de la décennie, voir Connelly (2004). 
26. «I always have lived on impulse [...]» (« He Lives His Impulses, Modern Screen, septembre 1936, p. 36-37).

27. Expression qui désigne un homme sauvage, brutal, rustre, etc. Au début de la décennie, Cagney et Gable sont ainsi qualifiés de «movie cavemen " (Motion Picture, mai 1932, p. 56).

28. "He's a rustic individual [...] He's rough-looking, [...] and a white tie and tails wouldn't be very becoming. » (Photoplay, juin 1938 p. 8-9).

29. Modern Screen, janvier 1938, p. 16.

30. " "As for clothes," sighed Mrs. T., "well, just any old coat and any old pair of trousers are all right for Spencer - whether they have any reason for being worn together or not." » (Modern Screen, mai 1940, p. 77-78).

31. «Laughter too... as Clark makes Spencer act as Myrna's stand-in! Spencer's willing but not able... if you get what we mean. » (Photoplay, mai 1938, p. 2).

32. "He's homely, too - they wouldn't waste a foot of celluloid on his profile alone because he isn't worth it. The close-up they make of him isn't meant to be a pretty picture - it's to show his tender eyes that are so transparent you can see his soul. » (Photoplay, juin 1938, p. 8-9).

33. "He's [...] sweet and sentimental. [...] His great capacity for sympathetic understanding completely envelops you » (Ibid.).

34. «[...] to a woman he represents the kind, the tender, the understanding man, and the type who would not fail her in a crisis. Every woman wants security, and there is about the Tracy personality that which promises complete loyalty and unwavering devotion as well as a bulwark of protection » (Motion Picture, mai 1941, p. 75).

35. Motion Picture, décembre 1936, p. 33, 66-67.

36. Voir par exemple : Modern Screen, décembre 1935, p. 29 ; août 1938, p. 88.

37. La redéfinition/revalorisation du mariage que l'on trouve par exemple dans les screwball comedies produites à partir du milieu de la décennie, peut être vue comme une réponse à une « crise du mariage » qui date d'avant la Dépression et que celle-ci ne fait donc qu'attiser (Shumway 1991: 8, Glitre 2006: 45-48). Parmi les représentations idéalisées de la famille qui se multiplient durant la seconde moitié des années 1930, voir notamment la série des Andy Hardy, initiée avec A Family Affair (George B. Seitz, 1937).

38. Voir par exemple Motion Picture, décembre 1936, p. 33; Modern Screen, août 1938, p. 33 ; et surtout Screenland, novembre 1939, p. 38-39; mars 1940 p. 34 ; Modern Screen, mai 1940, p. 28-29.

39. "Take a census of the women workers in the studios where Tracy does his stuff, and you'll hear things like: "Spencer Tracy is the finest, truest, squarest, cleanest, most decent gentleman on the whole lot!" " (Motion Picture, décembre 1936, p. 67).

40. "The moral must be: "Stop on the way up." "

41. "Well, that's alright for lady soap bubbles, but you know, there's something in a man that makes him want to go ahead, makes him wanna see how big he can get without busting. »

42. "What you did once, you can do again. [...] Me, I'm gonna be around all the time. I'm gonna take care of you. I'm gonna see you don't get so big you burst. »

43. Modern Screen, août 1938, p. 88-89. 
44. «McMasters is a wildcatter. If it wasn't for automobiles, he'd be driving a covered wagon. It's always been his breed that has opened up the country and made it what it is. So now I'm wondering if it gets to be out of line, in these United States, for a man like him to make a million dollars with his brains and or with his hands? Because if that's true, then we'd better rewrite this land-of-opportunity stuff. »

45. Photoplay, mars 1938, p.91-92. Voir également "Spence's charities", Hollywood, septembre 1937, p. 12.

46. Modern Screen, novembre 1938, p. 95.

47. « When he played Manuel, the tenderness was not acting only. It sprang from a natural source » (Ibid.).

48. Motion Picture, novembre 1937, p. 31.

49. Voir par exemple Photoplay, avril 1937, p. 57, 105 ; et surtout l'article « Because She Loved Him So Much ", Modern Screen, août 1938, p. 32-33, 88-89.

50. Voir notamment Modern Screen, septembre 1939, p. 36 ; Screenland, juillet 1940, p. 30-31, 76-77.

51. On peut également citer sa relation avec Franchot Tone dans They Gave Him a Gun (W. S. Van Dyke, 1937) ou avec le jeune homme qu'il prend sous son aile dans Edison, the Man.

52. «[...] a core of sweetness under his rugged strength » (Modern Screen, octobre 1937, p. 104).

53. «He's a tremendous force, Blackie is. If he were only a force for good instead of evil.»

\section{RÉSUMÉS}

Après être devenu une star de premier plan grâce à ses performances dans Fury (1936) et San Francisco (1936), Spencer Tracy conforte sa popularité aux États-Unis en figurant dans des succès commerciaux et critiques tels que Captains Courageous (1937), Test Pilot (1938), Boys Town (1938) ou Boom Town (1940). Les sondages réalisés par Gallup en 1940 confirment qu'il est alors la star hollywoodienne la plus appréciée du public américain, loin devant Mickey Rooney, Clark Gable et Bette Davis, les trois stars les plus populaires après lui. Cet article tente d'éclairer les raisons de ce succès exceptionnel en replaçant l'image de Tracy dans le contexte idéologique états-unien de la seconde moitié des années 1930. Grâce à l'analyse des films de l'acteur et des articles que lui consacrent les magazines, je mets en lumière la complexité de sa persona, en portant une attention particulière aux dimensions nationale et genrée de cette dernière. Il s'agit notamment de montrer que l'image de Tracy articule alors deux conceptions de la masculinité américaine, généralement distinguées en anglais par les termes de «manhood» et de «masculinity ", et résout ainsi une contradiction idéologique exacerbée par le processus de reconstruction nationale qui fait suite aux années de Dépression.

After becoming a major star thanks to his performances in Fury (1936) and San Francisco (1936), Spencer Tracy consolidated his popularity in the US by starring in critical and commercial 
successes such as Captain Courageous (1937), Test Pilot (1938), Boys Town (1938) or Boom Town (1940). The polls made by Gallup in 1940 confirmed that he was at the time the most loved Hollywood star in the US, far ahead of Mickey Rooney, Clark Gable and Bette Davis, the three most popular stars after him. This article attempts to understand the reasons for this exceptional success, by shedding light on Tracy's image in the US ideological context of the second half of the 1930s. Analyzing the actor's films and magazine articles about him, I highlight the complexity of his persona, particularly it's national and gendered facets. I aim to show that Tracy's image thus articulates two conceptions of American masculinity, usually distinguished in english by the terms "manhood" and "masculinity," and thereby resolves an ideological contradiction exacerbated by the process of national reconstruction that followed the years of the Great Depression.

INDEX

Mots-clés : masculinité, star studies, persona, États-Unis, Grande Dépression, cinéma, Tracy (Spencer)

Keywords : masculinity, star studies, persona, United States, Great Depression, cinema, Tracy (Spencer)

\section{AUTEUR}

\section{JULES SANDEAU}

Université Paul-Valéry-Montpellier 